\title{
Cerebrospinal fluid anandamide levels, cannabis use and psychotic-like symptoms
}

Celia J. A. Morgan, Emma Page, Carola Schaefer, Katharine Chatten, Amod Manocha, Sumit Gulati, H. Valerie Curran, Brigitta Brandner and F. Markus Leweke

\section{Summary}

Anandamide is a ligand of the endocannabinoid system. Animals show a depletion following repeated $\Delta^{9}$-tetrahydrocannabinol (THC) administration but the effect of cannabis use on central nervous system levels of endocannabinoids has not been previously examined in humans. Cerebrospinal fluid (CSF) levels of the endocannabinoids anandamide, 2-arachidonoylglycerol (2-AG) and related lipids were tested in 33 volunteers (20 cannabis users). Lower levels of CSF anandamide and higher levels of 2-AG in serum were observed in frequent compared with infrequent cannabis users. Levels of CSF anandamide were negatively correlated with persisting psychotic symptoms when drug-free. Higher levels of anandamide are associated with a lower risk of psychotic symptoms following cannabis use.

\section{Declaration of interest}

\section{None.}

The endocannabinoid system is emerging as crucial to many regulatory functions in the brain. The known endocannabinoids are anandamide, 2-arachidonoylglycerol (2-AG), oleoylethanolamide (OEA) and palmitoylethanolamide (PEA). Anandamide levels have been found to be elevated in the cerebrospinal fluid (CSF) of patients with antipsychotic-naive first-break schizophrenic psychosis ${ }^{1,2}$ and in the initial prodromal states of psychosis. ${ }^{3}$ This elevation is reversed by treatment with typical, but not atypical, antipsychotics, ${ }^{2}$ leading to the suggestion that anandamide has an adaptive, if not protective, role in psychosis and has a homeostatic role in controlling, for example, excessive dopamine release. ${ }^{4}$

Repeated treatment with $\Delta^{9}$-tetrahydrocannabinol (THC), the major psychotomimetic compound of cannabis, has been shown to downregulate anandamide signalling in the central nervous system (CNS) of rats (see for example Di Marzo et $a l^{5}$ ). Additionally, in patients with schizophrenia, cannabis use was shown to reduce the elevation in anandamide levels in CSF, when compared with patients who had never or only occasionally used cannabis. ${ }^{6}$ However, among healthy people who had not used cannabis significantly in their lives $(<5$ occasions in their lifetime) and those who were low-frequency users $(<50$ occasions in their lifetime) there were no differences in anandamide, suggesting that at least low-level cannabis use does not downregulate endocannabinoid signalling. No previous study had investigated levels of CSF endocannabinoids in heavy cannabis users and how these might relate to differences in the response to cannabis and associated risk of schizophrenia.

\section{Method}

Thirty-three individuals were recruited from a database created during previous studies. ${ }^{7}$ Individuals who used cannabis $>10$ times in a month were assigned to the 'heavy' use condition $(n=10)$, whereas individuals who used cannabis $<10$ times per month $(n=10)$ were assigned to the 'light' use condition. Controls were a group of 13 non-cannabis-using individuals. All controls had used cannabis less than eight times in their lifetime; two individuals had never used cannabis. All participants were free from any diagnosis of psychopathology or current medical problems according to DSM-IV criteria. ${ }^{8}$ All participants provided written, witnessed, informed consent. This study was approved by the National Research Ethics Committee for National Health Service research. Participants were compensated $\mathfrak{E} 10$ per hour (up to $£ 50$ ) for participation in the lumbar puncture procedure.

Participants were asked to abstain from recreational drugs and alcohol for $24 \mathrm{~h}$ before testing commenced. Each participant was tested at a single session lasting around $2 \mathrm{~h}$. Urine tests were also administered where available to assess the presence of THC and other substances. Drug use histories were taken and demographic data collected from participants. Lumbar punctures were conducted, with the participants choosing between the lying and sitting position. The needle was inserted in the lumbar 4-5 interspace. An atraumatic Sprotte needle was used to collect CSF. A sample of blood was taken from each participant. Blood samples were centrifuged for $10 \mathrm{~min}$ at $500 \mathrm{rpm}$. Samples were stored at $-80^{\circ} \mathrm{C}$ and then all samples were analysed at the Central Institute of Mental Health, Mannheim, Germany. To quantify fatty acid ethanolamides, $1 \mathrm{ml}$ aliquots from CSF and serum samples were spiked with $25 \mathrm{pmol}$ of $\left[{ }^{2} \mathrm{H}_{4}\right]$ anandamide, $\left[{ }^{2} \mathrm{H}_{4}\right] 2-\mathrm{AG}$, $\left[{ }^{2} \mathrm{H}_{4}\right]$ PEA and $\left[{ }^{2} \mathrm{H}_{4}\right]$ OEA to resemble the analytes and analyses were conducted by standard procedures reported elsewhere. ${ }^{2,4}$

Data from a previous study reported elsewhere ${ }^{7}$ indexed individual's acute psychotic response to naturalistically smoked cannabis on the Psychotomimetic States Inventory (PSI). ${ }^{9}$ These data were used for correlational analyses for all but three cannabis users who had not participated in the previous study. Nonparametric tests were used throughout because of non-normality of the data.

\section{Results}

The whole sample comprised 15 males and 18 females. One-way analysis of variance (ANOVA) showed no significant group differences in age $(22.1$ years, s.d. $=2.45)$ or years of education (16.5 years, s.d. $=1.99)$. There were no significant group differences in the number of regular alcohol users or level of alcohol use. Urine tests revealed the presence of THC in urine of all heavy cannabis users $(n=10 / 10)$, all but one light cannabis users $(n=9 / 10)$ and no controls $(n=0 / 13)$. One heavy cannabis user and one light cannabis user tested positive for MDMA (3,4-methylenedioxymethamphetamine).

The number of regular cannabis users and the number of years of cannabis use was not significantly different between the light 
(4.65 years, s.d. $=3.28)$ and heavy $(6.5$ years, s.d. $=2.92)$ groups. As expected, heavy users used cannabis on significantly more days per month (22.6 days, s.d. $=7.24)$ than light users (3.85 days, s.d. $=3.12)(F(1,16)=43.02, P<0.001)$.

A Kruskal-Wallis test results showed a trend-level group difference for anandamide levels in $\operatorname{CSF}\left(\chi^{2}=4.62, P=0.096\right)$ (Fig. $1)$. Orthogonal contrasts showed that heavy users had significantly lower CSF anandamide than light users (Mann-Whitney $U$-test $=$ 18.5, $P=0.015)$. There were no significant group differences for OEA, PEA or 2-AG in the CSF. A Kruskal-Wallis test showed a significant group difference $\left(\chi^{2}=13.669, P=0.001\right)$ in serum $2-A G$ levels (online Fig. DS1). A subsequent $U$-test indicated that heavy users had significantly higher serum 2-AG levels than controls $(U=3.000, P<0.001)$. There were no significant group differences for OEA, PEA or anandamide in serum. Cerebrospinal fluid anandamide and 2-AG did not correlate with serum. Among cannabis users, CSF anandamide correlated negatively with state psychotic symptoms when non-intoxicated $(r=-0.568, P=0.017)$.

\section{Discussion}

This was the first study to examine CSF eicosanoid levels in cannabis users. The study found lower CSF anandamide in heavy cannabis users compared with light cannabis users. Serum 2-AG levels were significantly higher in cannabis users than in controls. Cerebrospinal fluid anandamide correlated negatively with drugfree psychotic symptoms.

Preclinical work has suggested a downregulation of the endocannabinoid system ${ }^{5}$ following chronic THC administration in rats, which is partially supported by these findings. However, the current study extends this to suggest a possible upregulation of endocannabinoid signalling following moderate cannabis use, evident here in the elevated levels of CSF anandamide in light cannabis users but downregulation following heavy use. Control group anandamide levels were intermediate between the two groups, supporting this assertion.

We also observed a negative relationship between CSF anandamide levels and persistent psychotic symptoms. This is consistent with the findings of previous studies ${ }^{2}$ and is suggestive of a protective role of anandamide against psychotic-like symptoms, ${ }^{4}$ in particular negative symptoms. ${ }^{2,6}$ This may relate to the putative role of anandamide in stabilising dopamine release, given suggestions of increased dopamine release following smoking cannabis. ${ }^{10}$ Individuals with lower anandamide may be less able to modulate dopamine release, which increases psychotic-like symptoms both acutely and chronically.

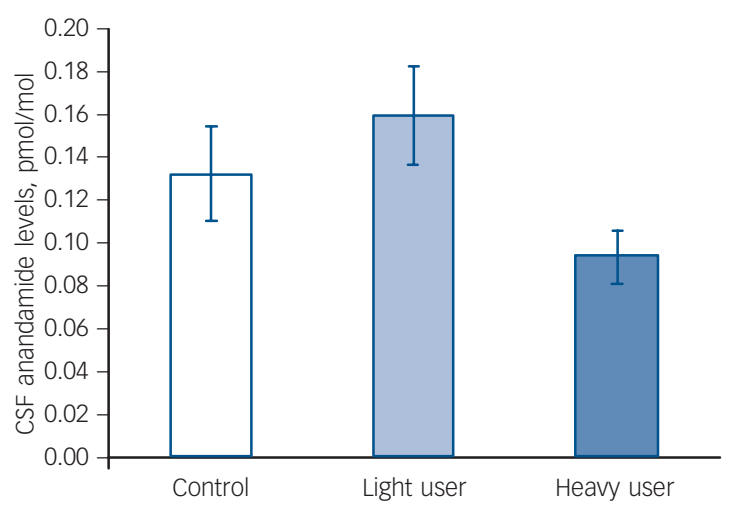

Fig. 1 Cerebrospinal fluid (CSF) anandamide levels across the three groups.
Serum levels of 2-AG were found to differ between heavy cannabis users and controls, with greater levels seen in cannabis users. This was an unexpected finding and, given the absence of correlation between serum and CSF levels, one that is difficult to interpret. Indeed, 2-AG is thought to be neuroprotective against excessive glutamate release and previous work has suggested a link between serum 2-AG and depression. ${ }^{11}$ However, the link between peripheral endocannabinoids and the CNS remains conjectural at present and warrants further research.

Our findings suggest that anandamide signalling is altered following cannabis use differentially in light and heavy users and psychotic-like symptoms are related to levels of this endocannabinoid in the brain. These findings suggest that healthy individuals who smoke cannabis and have lower levels of anandamide - either endogenously or through their cannabis use - may be more vulnerable to the acute and chronic psychotomimetic effects of cannabis, and therefore potentially more vulnerable to psychosis.

Celia J. A. Morgan, PhD, Emma Page, MSC, Clinical Psychopharmacology Unit, UCL, London, UK; Carola Schaefer, PhD, Department of Psychiatry and Psychotherapy, Central Institute of Mental Health, Medical Faculty Mannheim Heidelberg University, Mannheim, Germany; Katharine Chatten, MD, MRCA, Amod Manocha, MD, FRCA, Sumit Gulati, MD, FRCA, Centre for Anaesthesia, UCL

Hospitals Trust, London, UK; H. Valerie Curran, PhD, Clinical Psychopharmacology Unit, UCL, London, UK; Brigitta Brandner, MD, FRCA, Centre for Anaesthesia, UCL Hospitals Trust, London, UK; F. Markus Leweke, MD, Department of Psychiatry and Psychotherapy, Central Institute of Mental Health, Medical Faculty Mannheim, Heidelberg University, Mannheim, Germany

Correspondence: Celia Morgan, Clinical Psychopharmacology Unit, University College London, Gower St, London WC1E 6BT, UK. Email: c.morgan@ucl.ac.uk

First received 18 Sep 2012, final revision 4 Jan 2013, accepted 6 Feb 2013

\section{Funding}

This work was supported by an ECNP Research Grant for Young Scientists to C.J.A.M.

\section{References}

1 Leweke FM, Giuffrida A, Wurster U, Emrich HM, Piomelli D. Elevated endogenous cannabinoids in schizophrenia. Neuroreport 1999; 10: 1665-9.

2 Giuffrida A, Leweke FM, Gerth CW, Schreiber D, Koethe D, Faulhaber J, et al. Cerebrospinal anandamide levels are elevated in acute schizophrenia and are inversely correlated with psychotic symptoms. Neuropsychopharmacology 2004; 29: 2108-14.

3 Koethe D, Giuffrida A, Schreiber D, Hellmich M, Schultze-Lutter F, Ruhrmann $\mathrm{S}$, et al. Anandamide elevation in cerebrospinal fluid in initial prodromal states of psychosis. Br J Psychiatry 2009; 194: 371-2.

4 Leweke FM. Anandamide dysfunction in prodromal and established psychosis. Curr Pharm Des 2012; 18: 5188-93.

5 Di Marzo V, Berrendero F, Bisogno T, Gonzalez S, Cavaliere P, Romero J, et al. Enhancement of anandamide formation in the limbic forebrain and reduction of endocannabinoid contents in the striatum of delta9-tetrahydrocannabinoltolerant rats. J Neurochem 2000; 74: 1627-35.

6 Leweke FM, Giuffrida A, Koethe D, Schreiber D, Nolden BM, Neatby MA, et al. Anandamide levels in cerebrospinal fluid of first-episode schizophrenic patients: impact of cannabis use. Schizophr Res 2007; 94: 29-36.

7 Morgan CJA, Schafer G, Freeman TP, Curran HV. Impact of cannabidiol on the acute memory and psychotomimetic effects of smoked cannabis: naturalistic study. Br J Psychiatry 2010; 197: 285-90.

8 American Psychiatric Association. Diagnostic and Statistical Manual of Mental Disorders (4th edn) (DSM-IV). APA, 1994.

9 Mason OJ, Morgan CJ, Stefanovic A, Curran HV. The psychotomimetic states inventory (PSI): measuring psychotic-type experiences from ketamine and cannabis. Schizophr Res 2008; 103: 138-42.

10 Bossong MG, van Berckel BN, Boellaard R, Zuurman L, Schuit RC, Windhorst $A D$, et al. Delta 9-tetrahydrocannabinol induces dopamine release in the human striatum. Neuropsychopharmacology 2009; 34: 759-66.

11 Hill MN, Miller GE, Ho WS, Gorzalka BB, Hillard CJ. Serum endocannabinoid content is altered in females with depressive disorders: a preliminary report. Pharmacopsychiatry 2008; 41: 48-53. 